\title{
Cryo-EM Workflow Optimization
}

\author{
Maarten Kuijper ${ }^{1}$, Merijn de Jonge ${ }^{1}$, John Mitchels ${ }^{2}$, Stan Konings ${ }^{1}$, Ondrej Shanel ${ }^{2}$ and Rob van der \\ Ploeg ${ }^{1}$ \\ 1. Thermo Fisher Scientific, Materials \& Structural Analysis, Eindhoven, the Netherlands. \\ 2. Thermo Fisher Scientific, Materials \& Structural Analysis, Brno, Czech Republic.
}

Structural biologists have made great progress in discovering the structures of individual proteins and protein complexes. Much of the recent progress in the structural biology field stems from advances in cryo-transmission electron microscopy (cryo-TEM), in particular single particle analysis, which has made the elucidation of proteins and protein complexes at (near) atomic resolution almost routine. The key technological advances enabling this progress have been the maturation of cryo-TEM platforms (Thermo Scientific Krios and Thermo Scientific Talos Arctica), the revolution in image processing software the development of (electron counting) direct electron detectors and the advances made in the full automation of microscopy settings and data collection $[1,2,3,4]$.

Typically over multiple iterations, the biochemistry and vitrification conditions first need to be optimized before high resolution data can be acquired successfully. Thermo Fisher Scientific is preparing to launch several new systems, both EM tools and applications, that optimize the CryoEM workflow efficiency in those phases. These systems have new, better or faster integrated components increasing throughput or customer experience, but also enable cost effective distribution of workload over dedicated systems per workflow step. Special attention is given to establishing a seamless and hassle-free connectivity of the sample and data between those workflow components.

Recently, Thermo Fisher Scientific introduced the Thermo Scientific Glacios 200kV X-FEG system which is well suited to offload the Krios or Talos Arctica from the screening work to find the optimal biochemistry and vitrification settings. With a contamination free sample connectivity between the Glacios and the high resolution Talos Arctica/Krios CryoEM tool, the same sample can now be analyzed further for high resolution data collection for single particle analysis later.

The same seamless sample and data connectivity approach is introduced for the Cryo-lamella tomography workflow too where a biological sample is first targeted using correlative cryo light microscopy, then thinned by FIB in the Thermo Scientific Aquilos and then transferred with minimal contamination to the Krios for guided high-resolution tomography analysis of the lamella by using the stored coordinates of the lamella.

Considering the flow of the sample and the data from the sample preparation steps up to the imaging and data analysis steps is increasing the success rate and improving the efficiency of the CryoEM workflows. Although very important for today's sample and data connectivity, this is only the beginning of what we envision to be possible. Next generations of CryoEM platforms will be built upon this data centric approach, opening a wealth of possibilities for the future. In this presentation we discuss the future of sample and data connectivity focusing on optimizing the CryoEM workflows. 
References:

[1] The Nobel Prize in Chemistry 2017, Jacques Dubochet, Joachim Frank, Richard Henderson.

[2] Nature Methods, Special feature: Method of the Year 2015 (2015).

[3] K. R. Vinothkumar and R. Henderson, Quarterly Reviews of Biophysics (2016).

[4] Andreas Boland, Leifu Chang, David Barford, The potential of cryo-electron microscopy for structure-based drug design, Essays in Biochemistry (2017).

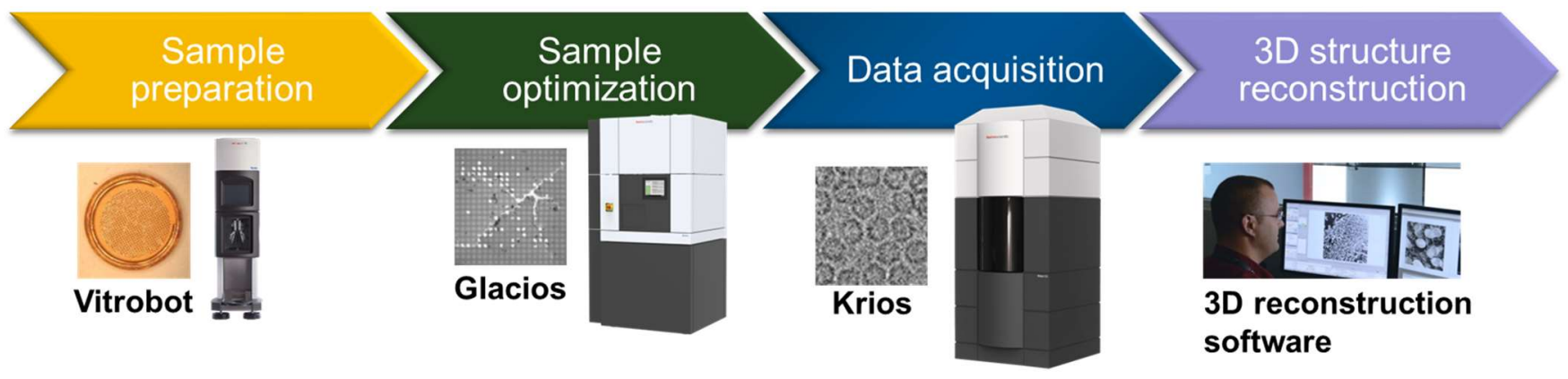

Figure 1. Describes the single particle analysis workflow from the sample preparation phase till data analysis phase. Typically over multiple iterations, the biochemistry and vitrification conditions are optimized before first high resolution data can be acquired. 